\title{
Comparação tecnológica, mineralógica e química da Formação Corumbataí em dois polos cerâmicos distintos visando sua aplicação na indústria de revestimentos cerâmicos
}

\section{(Technological, mineralogical and chemical comparison of the Corumbataí Formation in two distinct ceramic clusters for applications in ceramic tile industry)}

\author{
S. R. Christofoletti ${ }^{1}$, M. M. T. Moreno ${ }^{2}$ \\ ${ }^{1}$ Instituto Florestal, FEENA, Secretaria do Meio Ambiente, SP, Av. Navarro de Andrade, s/n, 13500-970 \\ ${ }^{2}$ Instituto de Geociências e Ciências Exatas, Unesp, Rio Claro, SP \\ sergioricardoc@gmail.com,mmoreno@rc.unesp.br
}

Resumo

\begin{abstract}
No presente trabalho foram estudadas as variações tecnológicas, químicas, mineralógicas e cerâmicas da Formação Corumbataí em três seções geológicas de dois polos cerâmicos distintos no estado de S. Paulo, Tambaú/Porto Ferreira (T/PF) e Santa Gertrudes (SG), com intuito de entender as variações que ocorrem nestas matérias primas buscando a diversificação de produtos. Para estudar estas variações, a metodologia empregada constou do levantamento de seções geológicas, análise mineralógica por difração de raios X na sua constituição total e menor que $2 \mu \mathrm{m}$, caracterização geoquímica para identificação dos principais elementos por ICP OES/MS e caracterização cerâmica para revestimento. Os resultados mineralógicos e químicos encontrados refletiram diretamente nos característicos cerâmicos obtidos. O sódio, elemento fundente encontrado principalmente no feldspato sódico, é mais abundante nas seções do Polo SG do que nas seções do PoloT/PF; este elemento age durante a sinterização em conjunto com o K das illitas, porém no Polo T/PF o conteúdo de feldspato potássico é maior que em SG; sendo assim, o K disponível pode ser menor. Além dos argilominerais do grupo illita, presente em todas as amostras, foram identificados argilominerais do grupo das esmectitas e caulinitas. De acordo com os resultados dos ensaios cerâmicos, as amostras das seções P-4 e P-11 do Polo T/PF foram classificadas dentro do grupo de absorção de água como BIII (poroso), sendo sua aplicação mais usual na produção de revestimentos com alta porosidade, azulejos e pastilhas, ou na formulação de revestimento pelo processo via seca e via úmida. Já nas amostras da seção P-19 do Polo SG, os resultados obtidos enquadraram-se dentro do grupo BIb, BIIb e BIII possibilitando sua aplicação, principalmente no caso do BIb e BIIb, na produção de revestimento pelo processo via seca ou na composição de massas para a produção de porcelanatos por via úmida e porosos. Estes resultados diferentes para as seções estudadas refletem diretamente na presença do tipo do feldspato presente, na composição dos óxidos principais, na granulometria e nas diferentes litologias encontradas nas seções geológicas.
\end{abstract}

Palavras-chave: Formação Corumbataí, cerâmica, argila.

Abstract

The technological, chemical, mineralogical and ceramicvariations of Corumbatai Formation in three geological sections of two different ceramic clusters in the State of S. Paulo, Tambaú/Porto Ferreira (T/PF) and Santa Gertrudes (SG), were studied,aiming to understand the variations that occur in these raw materials for product diversification. To study these variations, the methodology consisted of collection of geological sections, mineralogical analysis by X-ray diffraction in their full constitution and less than $2 \mu \mathrm{m}$ fraction, geochemical characterization for identification of major elements by ICP OES/MS, and ceramic tests for tiles. The chemical and mineralogical results directly influenced the ceramic characteristics. Sodium, which decreases the firing temperature and is found primarily in the sodic feldspar, is more abundant in the sections of Cluster SG than in sections of Cluster T/PF; this element acts during sintering together with the $K$ of illites, but the potassic feldspar content is higher in the Cluster T/PF than in the SG; therefore, the available K can be lower. In addition to the clay minerals of the illite group, present in all samples, clay minerals of the smectite and kaolinite groups were identified. According to the results of ceramic tests, samples of the P-4 and P-11 sections of Cluster T/PF were classified as waterabsorbing group BIII (porous), and their most common application is the production of tiles with high porosity, or in tile formulationsfor dry and wet processes. Considering the samples of P-19 section of Cluster SG, the results were classified in groups BIb, BIIb and BIII enabling their application, particularly in the case of BIb and BIIb, for the tile production by dry process, or in mass compositions for the production of porcelain stoneware by wet route and porous bodies. These different results for the studied sections were directly affected by the type of feldspar, composition of main oxides, particle size, and different lithologies found in the geological sections.

Kewwords: Corumbatai Formation, ceramic, clay. 


\section{INTRODUÇÃO}

O presente trabalho mostra as variações tecnológicas, mineralógicas e químicas que ocorrem entre a mesma unidade geológica em dois polos cerâmicos distintos no estado de S. Paulo. Foram levantadas três seções geológicas localizadas nos municípios de Araras, Porto Ferreira e Tambaú, sendo ambas fornecedoras de matéria prima para a indústria de revestimentos cerâmicos de dois importantes polos industriais. Os municípios de Porto Ferreira e Tambaú representam importantes municípios produtores de cerâmica estrutural focados principalmente na produção de blocos, telhas, pastilhas e no fornecimento de matéria prima para compor massas na produção de revestimento. Já a seção estudada na cidade de Araras representa uma importante mineração fornecedora de matéria prima para o polo cerâmico de Santa Gertrudes, sendo considerado o segundo maior do mundo na produção de revestimento cerâmico. Dados atuais mostram que o setor de revestimento cresce anualmente, tendo sido produzidos 1084,1 milhões $/ \mathrm{m}^{2}$ em 2014; o polo cerâmico de Santa Gertrudes responde por $65 \%$ da produção nacional, sendo que no ano de 2014 atingiu 575,58 milhões de $\mathrm{m}^{2}$. O Brasil situa-se como o $2^{\circ}$ maior produtor e consumidor do mundo, sendo a produção $73 \%$ por via seca, processo majoritário em Santa Gertrudes, e $27 \%$ por via úmida. O Polo de Santa Gertrudes domina a tecnologia pelo processo via seco, sendo atualmente o maior polo produtor; porém, observa-se uma tendência de aumento na produção

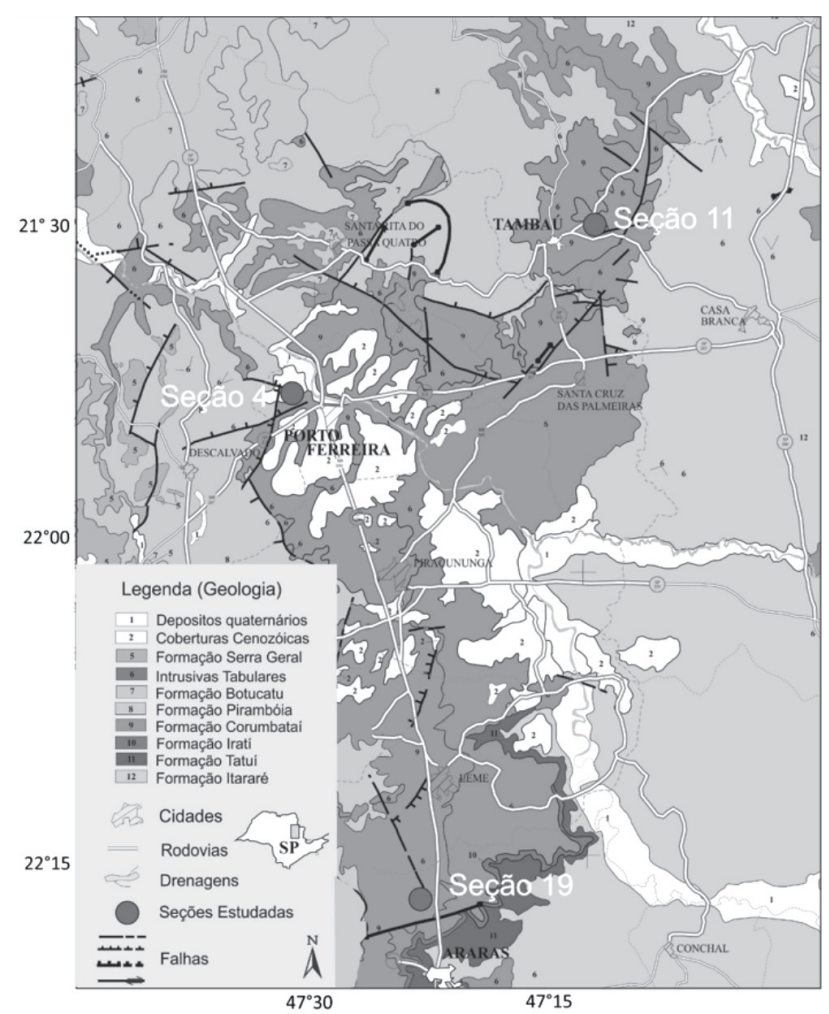

Figura 1: Mapa geológico da área de estudo incluindo os pontos estudados. Modificado de [5].

[Figure 1: Geological map of the study area including the studied points. Modified from [5].]

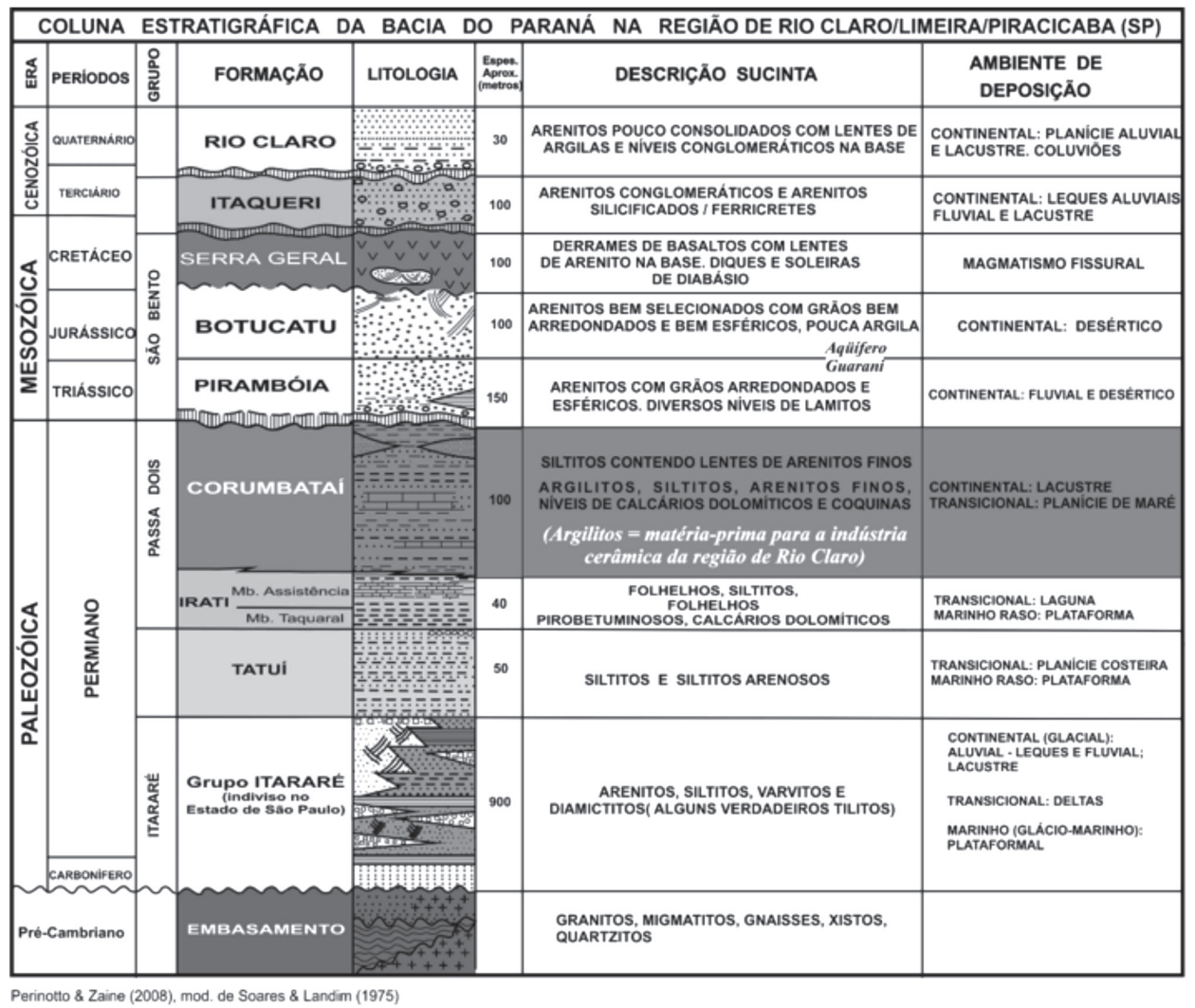

Figura 2: Coluna estratigráfica da Bacia do Paraná na região da área de estudo [6].

[Figure 2: Stratigraphic column of the Paraná Basin in the region of the study area [6].] 
de revestimentos pelo processo via úmida, em especial o porcelanato. Santa Gertrudes destaca-se no cenário nacional e internacional pelo baixo custo na produção do revestimento pelo processo via seca, sendo utilizada uma única matéria prima da Formação Corumbataí. O maior desafio do polo cerâmico de Santa Gertrudes é produzir o porcelanato com a matéria prima da Formação Corumbataí. Alguns trabalhos retratam este assunto, destacando-se [1-4]. Com este intuito, o presente trabalho tem por objetivo comparar as diferenças tecnológicas, geológicas e geoquímicas existentes nesta unidade geológica com o objetivo de buscar a melhora e a diversificação dos produtos fabricados no polo cerâmico de Santa Gertrudes.

\section{Localização e contexto geológico regional no Estado}

A área de estudocompreende três pontos, P-4, P-11 e P-19, localizados na região central do estado de S. Paulo nos municípios de Araras, Porto Ferreira e Tambaú. O acesso aos pontos P-19 e P-4 se dá pela Rod. Anhanguera, SP 330, e ao ponto P-11 pela Rod. Padre Donizete, SP 332 (Fig. 1). Geologicamente, a área de estudo está inserida na bacia sedimentar do Paraná, composta pelas seguintes unidades geológicas: rochas do Subgrupo Itararé, do Grupo Passa Dois (Formação Irati e Corumbataí), rochas do Grupo S. Bento (Formação Piramboia e Formação Botucatu), rochas intrusivas básicas da Formação Serra Geral, e culminando com coberturas Cenozóicas recentes e sedimentos quaternários (Fig. 2). A Formação Corumbataí, unidade geológica alvo do estudo é constituída principalmente por fácies argilosas compostas por siltitos (maciços, laminados ou intercalados), argilitos, folhelhos e arenitos finos a médios, de cores variadas, intercalados com siltitos arenosos ou argilosos [7]. Há algumas décadas, estas argilas constituem principal fonte de matéria prima para a indústria cerâmica, em especial para as indústrias de revestimento do polo cerâmico de Santa Gertrudes. Diversos autores [7-12] estudaram a Formação Corumbataí com destaque para estudos voltados para a caracterização geológica, tecnológica e geoquímica.

\section{MATERIAIS E MÉTODOS}

Foram realizados trabalhos de campo com o intuito de levantar seções geológicas e identificar as litofácies cerâmicas. Foram levantadas três seções geológicas verticais em minerações ativas denominadas P-4, P-11 e P-19, sendo as seções P-4 e P-11 do Polo Tambaú/Porto Ferreira (T/PF) e a seção P-19 do Polo Santa Gertrudes (SG). Posteriormente, foram coletadas amostras para a caracterização tecnológica, química e mineralógica.

A análise dos elementos principais foi feita por ICP-AES (espectrometria de emissão atômica com plasma acoplado indutivamente) e ICP-MS (espectrometria de massa com plasma acoplado indutivamente) das amostras fundidas com metaborato de lítio. A identificação das fases mineralógicas foi realizada por difração de raios X. A caracterização tecnológica foi feita em condições laboratoriais seguindo as normas daABNT [13]. As amostras inicialmente foram moídas em moinho de martelo, prensadas (foram obtidos corpos de prova de dimensões $7 \mathrm{~cm} \times 2 \mathrm{~cm}$ ) e queimadas a $1050{ }^{\circ} \mathrm{C}$ em forno de laboratório. Foram feitos os testes de absorção de água, porosidade aparente, densidade aparente, resistência à flexão, retração linear de queima e perda ao fogo. Também foi feita a análise granulométrica pela técnica de difração de laser no equipamento Mastersizer 2000 da Malvern, na qual uma alíquota da amostra moída foi colocada em uma solução de água com três gotas de hexametafosfato de sódio $(10 \%)$, aplicando $15 \mathrm{~s}$ de ultrassom com deslocamento ultrassônico de 12,5 conforme escala do equipamento.

\section{RESULTADOS E DISCUSSÃO}

\section{Análise geoquímica}

Os resultados da quantificação dos elementos principais na forma de óxidos são mostrados na Tabela I. Nestas matérias primas, por serem provenientes de rochas sedimentares silicáticas, o $\mathrm{SiO}_{2}$ é o mais abundante em todas as amostras das três seções estudadas, sendo que na seção P-19 do Polo SG obteve-se o maior valor médio de 67,23\% (Tabela I), uma vez que estes materiais, usados no polo SG foram menos expostos aos processos geológicos intempéricos. $\mathrm{O}$ teor de $\mathrm{Al}_{2} \mathrm{O}_{3}$ apresentou valores médios de $18,60 \%$ para as amostras da seção P-4, de 10,63\% para a seção P-11 e de $14,90 \%$ para a seção P-19, estando as maiores concentrações nas amostras da seção P-4 e na amostra 8 da seção P-19,indício da presença do argilomineral caulinita. $\mathrm{O}$ elemento sódio,considerado um importante fundente, apresentou valores médios na forma de óxido de sódio $\left(\mathrm{Na}_{2} \mathrm{O}\right)$ de $0,33 \%$ para a seção $\mathrm{P}-4,0,75 \%$ para a seção P-11 e 3,21\% para a seção P-19. A porcentagem maior deste óxido nas amostras da seção P-19 (Polo SG) é resultante da presença nestas argilas do feldspato do tipo albita [12]. Já $\mathrm{K}_{2} \mathrm{O}$ representa um importante óxido fundente no processo de fabricação via seca, onde as temperaturas de queima são em $1100{ }^{\circ} \mathrm{C}$ ou menores, sendo encontrado nos argilominerais do grupo das illitas $\left(\mathrm{K}, \mathrm{H}_{3} \mathrm{O}\right) .(\mathrm{Al}, \mathrm{Mg}, \mathrm{Fe})_{2}(\mathrm{Si}, \mathrm{Al})_{4} \mathrm{O}_{10}\left[(\mathrm{OH})_{2}, \mathrm{H}_{2} \mathrm{O}\right]$. $\mathrm{A}$ presença de outros minerais contendo $\mathrm{K}$, como micas, zeólitas e principalmente feldspatos $\left(\mathrm{KAlSi}_{3} \mathrm{O}_{8}\right)$, não permite uma avaliação direta como fundente unicamente a partir da análise química, uma vez que este mineral é mais resistente à decomposição com a temperatura do que os feldspatos sódicos. $\mathrm{Fe}_{2} \mathrm{O}_{3}$, encontrado principalmente no mineral hematita e na composição química dos argilominerais, apresentou sua maior concentração nas amostras da seção P-4 com valor médio $6,33 \%$. Em relação ao $\mathrm{CaO}$, as amostras da seção P-11 apresentaram as maiores porcentagens, com valor médio 5,84\%, que é um indício da presença de carbonatos (calcita e/ou dolomita) nas amostras da seção.

\section{Análise mineralógica}

A mineralogia foi determinada por difratometria de raios $\mathrm{X}$, onde se identificaramosseguintes grupos: óxidos, carbonatos, silicatos "tectossilicatos" e os filossilicatos 
Tabela I - Resultados de análises químicas dos principaiselementos (em \% em peso de óxido).

[Table I - Results of chemical analyzes of the major elements (in wt.\% of oxide).]

\begin{tabular}{lccccccccccc}
\hline \multicolumn{1}{c}{ Amostras } & $\mathbf{A l}_{\mathbf{2}} \mathbf{O}_{\mathbf{3}}$ & $\mathbf{C a O}$ & $\mathbf{T i O}_{\mathbf{2}}$ & $\mathbf{S i O}_{\mathbf{2}}$ & $\mathbf{F e}_{\mathbf{2}} \mathbf{O}_{\mathbf{3}}$ & $\mathbf{K}_{\mathbf{2}} \mathbf{O}$ & $\mathbf{M g O}$ & $\mathbf{M n O}$ & $\mathbf{N a}_{\mathbf{2}} \mathbf{O}$ & $\mathbf{P}_{\mathbf{2}} \mathbf{O}_{\mathbf{5}}$ & $\mathbf{L O I}$ \\
\hline P4-Am-1 & 18,66 & 0,04 & 0,89 & 58,53 & 6,76 & 1,63 & 2,62 & 0,18 & 0,04 & 0,03 & 6,92 \\
P4-Am-2 & 17,35 & 0,11 & 0,72 & 62,52 & 5,10 & 3,00 & 1,33 & 0,10 & 0,88 & 0,04 & 5,07 \\
P4-Am-3 & 19,81 & 0,01 & 0,89 & 57,73 & 7,12 & 1,53 & 0,61 & 0,07 & 0,07 & 0,15 & 7,05 \\
MÉDIA & $\mathbf{1 8 , 6 0}$ & $\mathbf{0 , 0 5}$ & $\mathbf{0 , 8 3}$ & $\mathbf{5 9 , 6 0}$ & $\mathbf{6 , 3 3}$ & $\mathbf{2 , 0}$ & $\mathbf{1 , 5 2}$ & $\mathbf{0 , 1 1}$ & $\mathbf{0 , 3 3}$ & $\mathbf{0 , 0 7}$ & $\mathbf{6 , 3 4}$ \\
P11-Am-1 & 11,28 & 2,32 & 0,48 & 65,10 & 3,22 & 5,69 & 2,24 & 0,03 & 1,01 & 0,22 & 3,84 \\
P11-Am-2 & 9,83 & 8,81 & 0,37 & 51,73 & 3,19 & 5,58 & 4,84 & 0,10 & 0,69 & 0,09 & 12,02 \\
P11-Am-3 & 12,47 & 1,50 & 0,46 & 65,61 & 3,21 & 7,20 & 2,05 & 0,03 & 0,91 & 0,19 & 3,18 \\
P11-Am-4 & 12,56 & 0,47 & 0,50 & 64,65 & 4,08 & 6,96 & 2,50 & 0,05 & 0,84 & 0,10 & 2,73 \\
P11-Am-5 & 3,97 & 21,41 & 0,15 & 49,36 & 1,46 & 2,18 & 1,03 & 0,10 & 0,18 & 0,03 & 18,43 \\
P11-Am-6 & 13,71 & 0,57 & 0,51 & 64,24 & 3,75 & 8,14 & 2,16 & 0,04 & 0,88 & 0,13 & 2,31 \\
MÉDIA & $\mathbf{1 0 , 6 3}$ & $\mathbf{5 , 8 4}$ & $\mathbf{0 , 4 2}$ & $\mathbf{6 0 , 1 1}$ & $\mathbf{3 , 1 5}$ & $\mathbf{5 , 9 5}$ & $\mathbf{2 , 4 7}$ & $\mathbf{0 , 0 5}$ & $\mathbf{0 , 7 5}$ & $\mathbf{0 , 1 2}$ & $\mathbf{7 , 0 8}$ \\
P19-Am-1 & 14,81 & 0,79 & 0,58 & 67,28 & 5,15 & 3,91 & 2,03 & 0,03 & 2,84 & 0,11 & 1,94 \\
P19-Am-2 & 15,31 & 0,86 & 0,60 & 69,78 & 4,25 & 3,78 & 1,61 & $<0,01$ & 3,58 & 0,20 & 1,96 \\
P19-Am-3 & 15,35 & 1,94 & 0,55 & 66,74 & 4,59 & 3,68 & 2,63 & 0,08 & 4,08 & 0,14 & 2,25 \\
P19-Am-4 & 15,88 & 0,76 & 0,53 & 66,42 & 4,91 & 4,02 & 1,76 & 0,02 & 3,23 & 0,20 & 3,00 \\
P19-Am-5 & 13,50 & 1,95 & 0,42 & 67,81 & 3,73 & 2,77 & 1,84 & 0,03 & 3,93 & 0,13 & 2,72 \\
P19-Am-6 & 15,65 & 0,68 & 0,48 & 67,10 & 4,07 & 2,53 & 2,29 & 0,06 & 4,24 & 0,14 & 2,62 \\
P19-Am-7 & 11,51 & 5,20 & 0,42 & 61,07 & 3,45 & 2,12 & 3,77 & 0,08 & 3,70 & 0,16 & 6,04 \\
P19-Am-8 & 21,28 & 0,18 & 0,68 & 60,72 & 5,57 & 1,76 & 1,19 & $<0,01$ & $<0,01$ & 0,04 & 7,92 \\
P19-Am-9 & 10,88 & 0,22 & 0,48 & 77,56 & 3,21 & 1,73 & 1,15 & 0,11 & 0,06 & 0,10 & 4,04 \\
MÉDIA & $\mathbf{1 4 , 9 0}$ & $\mathbf{1 , 4 0}$ & $\mathbf{0 , 5 3}$ & $\mathbf{6 7 , 2 3}$ & $\mathbf{4 , 3 2}$ & $\mathbf{2 , 9 2}$ & $\mathbf{2 , 0 3}$ & $\mathbf{0 , 0 6}$ & $\mathbf{3 , 2 1}$ & $\mathbf{0 , 1 3}$ & $\mathbf{3 , 6 1}$ \\
\hline
\end{tabular}

LOI-perda ao fogo.
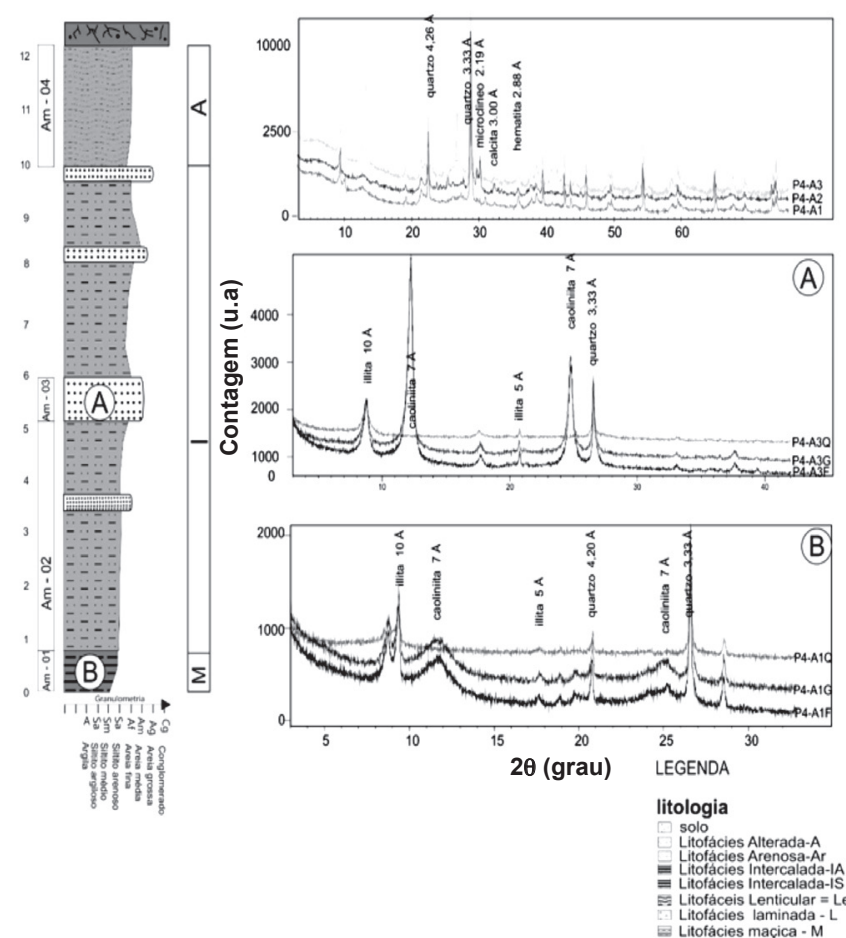

Figura 3: Seção geológica P-4 incluindo as litofácies e a mineralogia. [Figure 3:Geological section P-4 including lithofacies and mineralogy.]

representados pelos argilominerais. Entre os tectossilicatos se destacam o quartzo, sempre presente, e os feldspatos microclínio e albita (Figs. 3 a 5). O microclínio representa o feldspato potássico mais frequente nas amostras das seções P-4 e P-11 do Polo T/PF com seu pico principal d=3,24Å. Já nas

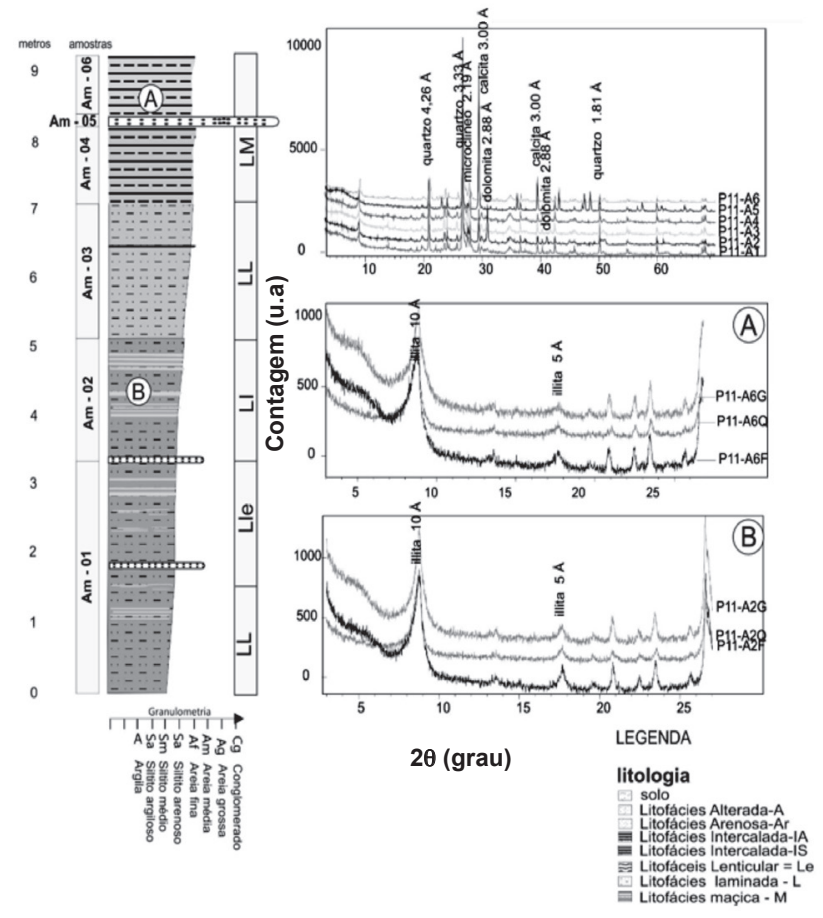

Figura 4: Seção geológica P-11 incluindo as litofácies e a mineralogia.

[Figure 4: Geological section P-11 including lithofacies and mineralogy.]

amostras da seção P-19 (Polo SG), o feldspato predominante é a albita e às vezes ocorre associado ao microclínio. Os carbonatos encontrados são representados principalmente pela calcita com $\mathrm{d}=3,00 \AA$ e pela dolomita com $\mathrm{d}=2,88 \AA$. 


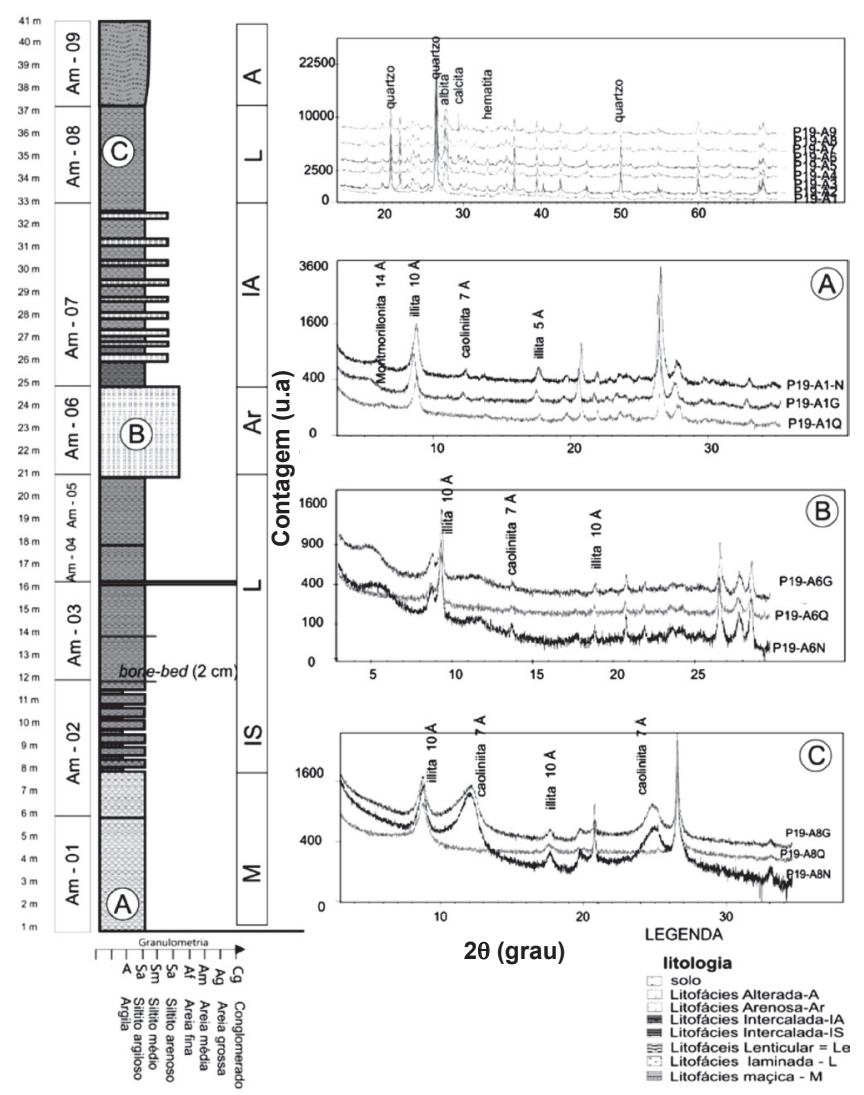

Figura 5: Seção geológica P-19 incluindo as litofáciese mineralogia. [Figure 5: Geological section P-19 includinglithofacies and mineralogy.]

O óxido identificado nas amostras estudadas é a hematita com $\mathrm{d}=2,69 \AA$, sendo encontrado em pequenas quantidades, com destaque para a seção P-4 com valor médio de 6,33\%, sendo caracterizado por um pico de alta intensidade no difratograma de raios X (Fig. 3). Já em relação aos argilominerais, a illita representa o predominante em todas as seções estudadas nos polos (SG e T/PF), sendo seguida pela caulinita encontrada em maiores quantidades nas amostras das porções superiores, em especial da seção P-4 e na seção P19-Amostra-8. Em relação aos minerais expansivos, observa-se baixa presença destes nas seções estudadas.

\section{Análise granulométrica}

A Fig. 6 mostra as curvas de distribuição de tamanho de partícula (frequência acumulada) das amostras das três seções.Os resultados das análises granulométricas foram agrupados, para fins comparativos, nos intervalos de diâmetro esférico equivalente (D.E.E.) de $0,01 \leq \phi \leq 1$, $1 \leq \phi \leq 10,10 \phi \leq 100$ e $100 \leq \phi \leq 1000 \mu \mathrm{m}$ (Fig. 7). As maiores concentrações das partículas das seções ficaram entre os intervalos $1-10 \mu \mathrm{m}$ e $10-100 \mu \mathrm{m}$ com valores médios, respectivamente, $36,21 \%$ e $53,02 \%$ para a seção P-4, $27,09 \%$ e $55,74 \%$ para a seção P- 11 e $36,00 \%$ e $57,10 \%$ para a seção P-19. Observou-se que no intervalo $0,01-1$ $\mu \mathrm{m}$ os valores médios para as seções foram de $\sim 5 \%$ (Fig.
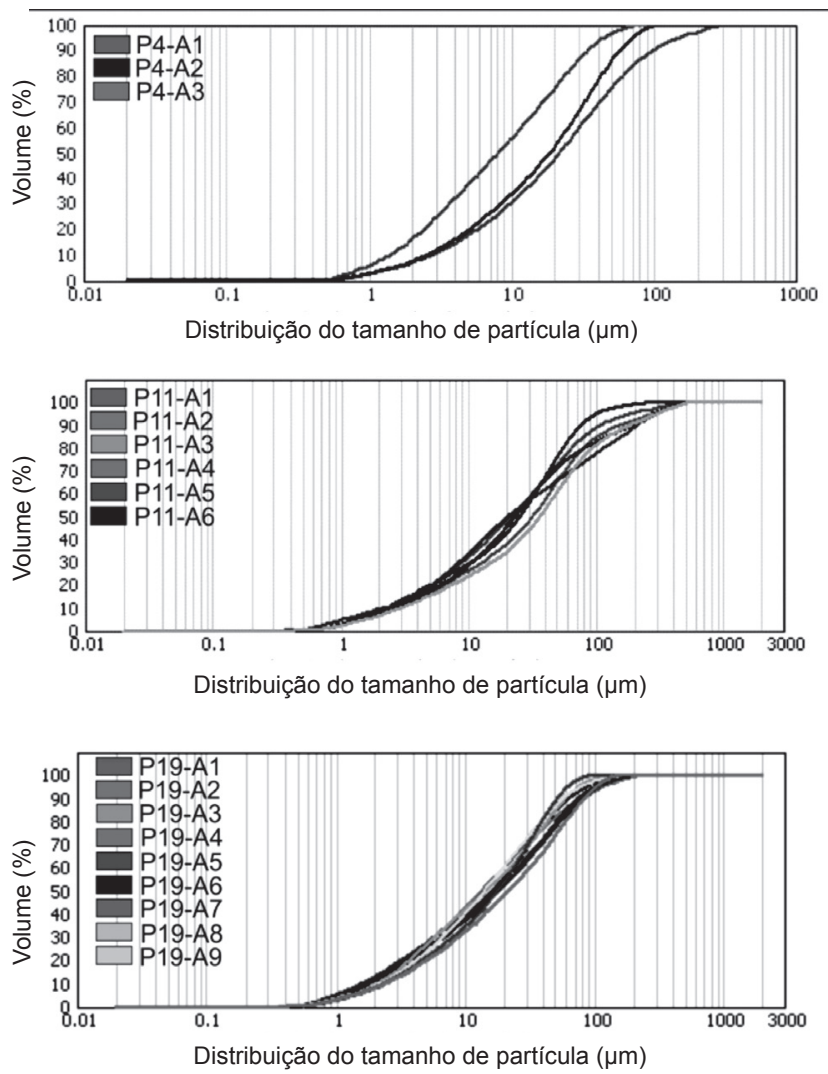

Figura 6: Curvas de distribuição granulométrica das amostras das seções estudadas.

[Figure 6:Particle size distribution curves of the samples of studied sections.]

7). Na faixa 1-10 $\mu \mathrm{m}$ as amostras das seções P-4 e P-19 apresentaram valores médios $36 \%$ e a amostra P-11 27\%. No intervalo $10-100 \mu \mathrm{m}$ as maiores concentrações das partículas estão nas amostras do Polo SG, seção P-19, com valor médio $57 \%$. No intervalo $100-1000 \mu \mathrm{m}$ as amostras da seção P-11 foram as que apresentaram o valor médio mais elevado atingindo $10,75 \%$, tendo apresentado em conjunto a distribuição granulométrica de moagem mais grossa. Observa-se uma tendência das amostras da base serem mais finas que as amostras das porções superiores das seções, devido aos processos intempéricos de hidrólise terem concentrado nestes níveis minerais mais resistentes, como quartzo, caulinita, carbonatos e feldspatos de potássio. A maior concentração de partículas na faixa granulométrica 100-1000 $\mu \mathrm{m}$ na seção P-11 pode ser resultante da presença elevada de carbonatos encontrados nos difratogramas de raios X (Fig. 4), o qual contribuiu também para os elevados valores de absorção de água (Tabela III). As amostras da seção P-19 apresentaram uma distribuição de partículas mais próxima entre si, resultando em um empacotamento semelhante das partículas durante o processo de prensagem.

\section{Caracterização tecnológica}

Os resultados dos ensaios cerâmicos apresentaramse diferentes para as amostras das seções P-4 e P-11 em 

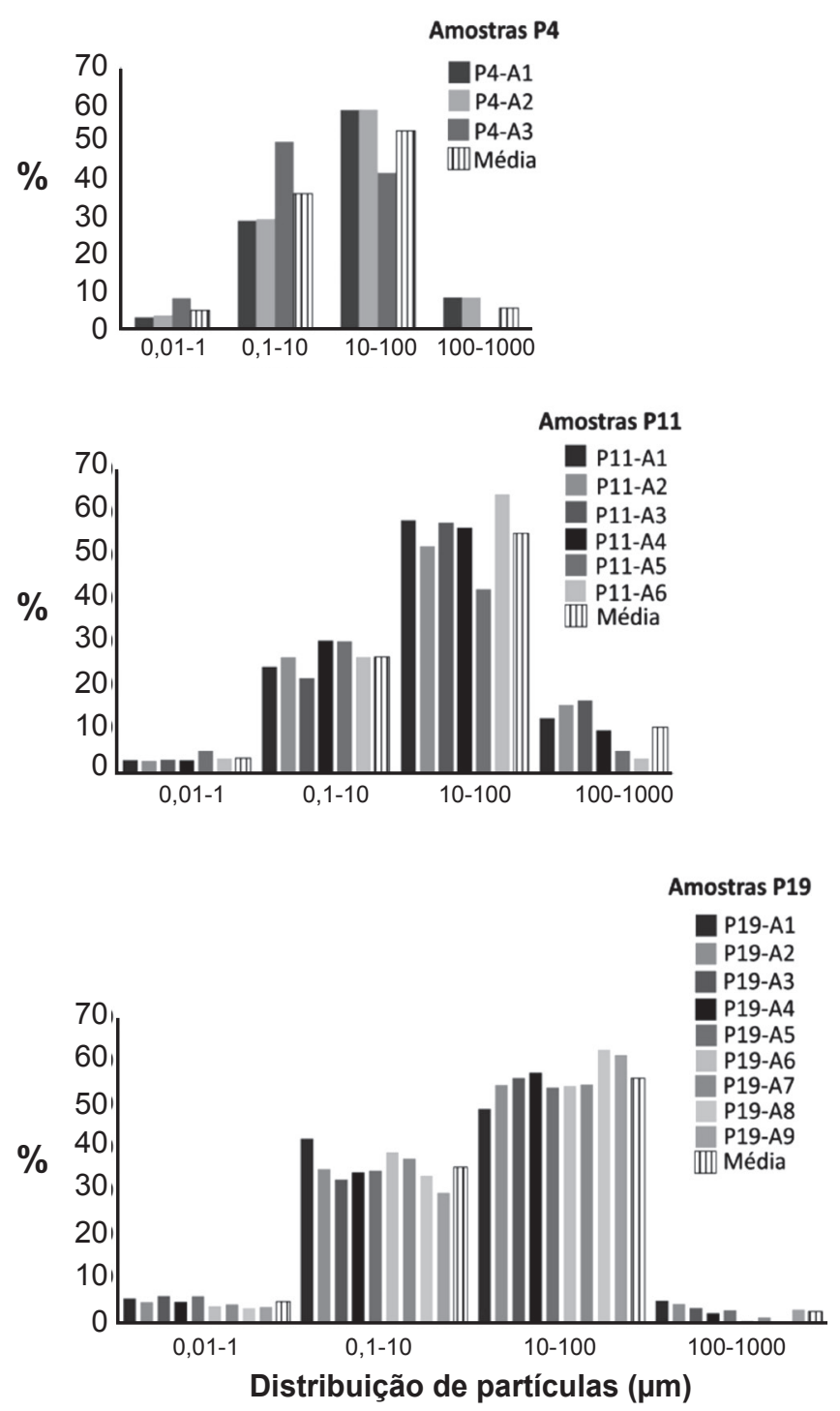

Figura 7: Histogramas dos resultados de análise granulométrica das amostras das seções estudadas.

[Figure 7: Histograms ofthe results of particle size analysis of the samples of studied sections.]

relação à seção P-19 (Tabela II). A maioria das amostras das seções P-4 e P-11 (Polo SG) enquadrou-se dentro do grupo de absorção de água BIII, com valores de absorção de água entre $10 \%$ e $20 \%$ e resistência mecânica entre 150 e $200 \mathrm{kgf} / \mathrm{cm}^{2}$, exceção feita à amostra P11-Amostra-4, que foi classificada no grupo BIIb (Tabela III). As amostras da seção P-19 apresentaram resultados mais adequados, segundo a classificação dos grupos de revestimentos cerâmicos, principalmente pelo maior conteúdo de $\mathrm{Na}_{2} \mathrm{O}$ (Tabela I), para serem utilizadas na indústria de revestimento cerâmico, sendo classificadas nos grupos BIa, BIb, BIIb e BIII, com uma amostra no BIa, duas no BIb, trêsno BIIb e três no BIII (Tabela II).

\section{CONCLUSÕES}

Foram observadas diferenças nas características quími- cas, mineralógicas, cerâmicas e granulométricas das seções estudadas nos Polos SG e T/PF. Estas diferenças refletem diretamente nas propriedades tecnológicas. De acordo com a mineralogia identificada por difração de raios X, o mineral quartzo encontra-se em todas as amostras. Observa-se também feldspatos microclínio e plagioclásio albita, além de calcita, dolomita e hematita. Dentre os argilominerais, a illita é predominante nas seções estudadas, exceção feita para as amostras P4-amostra 3 e P-19-amostra 8, nas quais o argilomineral caulinita é predominante. Observou-se a presença da montmorilonita na amostra P-19-amostra 1. Nas amostras das seções P-4 e P-11 (Polo T/PF), o feldspato predominante é o microclínio e nas amostras da seção P-19 (Polo SG) é a albita, contribuindo significativamente para a melhor sinterização das amostras, exceto nas três amostras do topo, nas quais, apesar da distribuição granulométrica ser semelhante e consequentemente o empacotamento, os teores de $\mathrm{Na}_{2} \mathrm{O}$ e $\mathrm{K}_{2} \mathrm{O}$ são baixo sem P-19-amostra 8 e P-19-amostra 9; em P-19-amostra 7, o conteúdo de carbonatos resultou em amostra mais porosa. Os carbonatos representados pelos minerais calcita e dolomita ocorrem com presença elevada nas amostras da seção P-11. A presença elevada do $\mathrm{K}_{2} \mathrm{O}$ nas amostras das seções P-4 e P-11 é resultante da presença do feldspato microclínio e da illita. Já a elevada presença do $\mathrm{Na}_{2} \mathrm{O}$ nas amostras do $\mathrm{P}-19$ deve-se à presença elevada do feldspato do tipo albita identificados nos difratogramas de raios X. Nas amostras das seções P-4 e P-11, observase uma presença mais elevada do argilomineral caulinita em relação às amostras das seções do P-15. Nas análises granulométricas,observou-se uma distribuição de partículas mais fina e homogênea entre as amostras da seção P-19 em relação às seções $\mathrm{P}-4 \mathrm{e} \mathrm{P}-11$. Este fato deve-se às seções (P-4 e P-11) no polo cerâmico T/PF, que estão localizadas nas porções mais superiores da Formação Corumbataí, onde as fácies cerâmicas são mais arenosas gerando uma distribuição de partículas mais grossas, enquanto que as amostras da seção P-19 são encontradas nas porções mais basais da Formação Corumbataí. Estas características refletiram diretamente nas propriedades cerâmicas permitindo classificar as amostras das seções P-4 e P-11 dentro do grupo de absorção de água BIII e da seção P-15 nos grupos BIb, BIIb e BIII. De acordo com o grupo de absorção de água, as amostras das seções P-4 e P-11 são classificadas comercialmente como porosas e as amostras da seção P-19 como porcelanato, grés e poroso. As seções estudadas no Polo T/PF representam a porção intermediária a superior da Formação Corumbataí, enquanto a seção do Polo SG abrange fácies basais superiores com uma espessura total de 41 metros. De acordo com os resultados cerâmicos das 9 amostras estudadas das seções P-4 e P-11 (Polo T/PF), 8 foram classificadas dentro do Grupo BIII, e das 9 amostras da seção P-19 (Polo SG), três foram classificadas dentro do Grupo BIII, sendo estas amostras localizadas na porção superior das seções estudadas. No estudo faciológico e de campo, identificou-se litofácies intercaladas arenosas nas porções superiores da Formação Corumbataí nos dois polos estudados, o que refletiu diretamente no processo de moagem, empacotamento e 
Tabela II - Resultados de ensaios tecnológicos cerâmicos (corpos de prova de $2 \mathrm{~cm}$ x $7 \mathrm{~cm}$ queimados em laboratório a $1050^{\circ} \mathrm{C}$ ): MRF - resistência a flexão, AA - absorção de água, PA - porosidade aparente, RLQ - retração linear de queima e GABS - grupo de absorção de água.

[Table II - Results of ceramic tests $\left(2 \mathrm{~cm} \times 7 \mathrm{~cm}\right.$ samples fired in laboratory at $\left.1050{ }^{\circ} \mathrm{C}\right):$ MRF - flexural strength, AA - water absorption, $P A$ - apparent porosity, RLQ - linear shrinkage afterfiring, and GABS - group of water absorption.]

\begin{tabular}{lccccc}
\hline Amostras & M.R.F $\left(\mathrm{kgf} / \mathrm{cm}^{2}\right)$ & AA $(\%)$ & PA (\%) & RLQ (\%) & GABS \\
\hline & \multicolumn{5}{c}{ Ponto 4} \\
P4-Am-1 & 244,40 & 14,70 & 28,41 & 3,97 & BIII \\
P4-Am-2 & 200,56 & 11,06 & 23,39 & 3,57 & BIII \\
P4-Am-3 & 168,95 & 17,94 & 32,90 & 2,54 & BIII \\
& \multicolumn{2}{c}{ Ponto 11} & & \\
P11-Am-1 & 140,00 & 8,61 & 17,86 & 2,88 & BIII \\
P11-Am-2 & 100,87 & 16,15 & 24,49 & 1,91 & BIII \\
P11-Am-3 & 110,02 & 9,89 & 19,98 & 3,24 & BIII \\
P11-Am-4 & 220,23 & 4,55 & 10,24 & 7,42 & BIIb \\
P11-Am-5 & 120,00 & 32,42 & 47,19 & --- & BIII \\
P11-Am-6 & 190,56 & 10,46 & 21,13 & 3,17 & BIII \\
& & & & \\
P19-Am-1 & 474,00 & 1,17 & 2,81 & 8,43 & BIb \\
P19-Am-2 & 461,91 & 1,37 & 3,24 & 8,87 & BIb \\
P19-Am-3 & 338,47 & 7,82 & 16,86 & 4,88 & BIIb \\
P19-Am-4 & 571,31 & 0,26 & 0,63 & 8,24 & BIa \\
P19-Am-5 & 273,77 & 8,74 & 18,29 & 5,31 & BIIb \\
P19-Am-6 & 299,19 & 6,43 & 14,14 & 7,94 & BIIb \\
P19-Am-7 & 146,69 & 18,27 & 33,44 & 0,40 & BIII \\
P19-Am-8 & 271,34 & 15,40 & 29,67 & 4,65 & BIII \\
P19-Am-9 & 86,98 & 17,47 & 31,54 & 0,28 & BIII \\
\hline
\end{tabular}

Tabela III - Classificação dos grupos de revestimentos cerâmicos, segundo ABNT [13]. [Table III - Classification of ceramic tile groups, according to ABNT [13].]

\begin{tabular}{cccc}
\hline Absorção de água (\%) & Resistência à flexão $\left(\mathrm{kgf} / \mathrm{cm}^{2}\right)$ & Grupo ABS & Nomenclatura comercial \\
\hline 0 a 0,5 & 300 a 500 & BIa & Porcelana \\
0,5 a 3,0 & 300 a 450 & BIb & Grés \\
3,0 a 6,0 & 320 a 350 & BIIa & Semi-grés \\
6,0 a 10,0 & 180 a 300 & BIIb & Semi-poroso \\
10,0 a 20,0 & 150 a 200 & BIII & Poroso \\
\hline
\end{tabular}

sinterização da peça durante a queima resultando na queda das propriedades cerâmicas. $\mathrm{O}$ conhecimento científico da Formação Corumbataí em diferentes locais é de importância para buscar a diversificação de produtos, ampliar as reservas existentes e melhora nos produtos atualmente fabricados.

\section{AGRADECIMENTOS}

Os autores agradecem o apoio financeiro da FAPESPFundação de Amparo à Pesquisa do Estado de S. Paulo, Proc.
2012/24219-9.

\section{REFERÊNCIAS}

[1] F.G. Melchiades, M.T. Daros, F.C. Zanelato, A.O. Boschi, "Viabilidade da fabricação de porcelanatos por via seca a partir de massas de cor de queima clara. Parte I: Condições de moagem e homogeneização da massa", Ceram. Ind. 17, 4 (2012) 13-21.

[2] F.G. Melchiades, L.R.S. Conserva, S. Nastri, E. Cabral, 
A.O. Boschi, "Viabilidade da fabricação de porcelanatos por via seca a partir de massas de cor de queima clara. Parte II: Condições de granulação da massa", Ceram. Ind.17, 5-6 (2012) 14-21.

[3] F.G. Melchiades, L.R.S. Conserva, S. Nastri, A.P. Leite, A.O. Boschi, "Viabilidade da fabricação de porcelanatos por via seca a partir de massas de cor de queima clara. Parte III: Composição da massa”,Ceram. Ind. 18, 1 (2013)18-24.

[4] A.C.A. Prado, C.D. Roveri, R.R. Rocha, A. Zanardo, M.M.T. Moreno, J.F.M. Motta, A.P. Menegazzo, "Rheology of clays from the Corumbataí Formation and their application in porcelain tile production", in QUALICER 9, Logui Impr., Castellón, Espanha 3(2006)245-249.

[5] CPRM, Projeto Mogi-Guaçu/Pardo, Carta Geológica Compilada, Secretaria do Meio Ambiente, S. Paulo, SP,3, (1998) $92 \mathrm{p}$.

[6] J.A.J. Perinotto, M.F. Zaine, "Patrimônios naturais e história geológica da região de Rio Claro-SP", Rev. Arq. Púb. Hist. Município de Rio Claro 1 (2008).

[7] S.R. Christofoletti, "Um modelo de classificação geológico-tecnológica das argilas da Formação Corumbataí utilizadas nas indústrias do Polo Cerâmico de Santa Gertrude", Tese Dr. Geociências, Inst. de Geoci. Ci. Ex., Univ. Est. Paulista, Rio Claro, SP (2003).

[8] S.H.M. Souza, "Fácies sedimentares das Formações Estrada Nova e Corumbataí no Estado de São Paulo. São Paulo", Diss. Mestrado Geologia Regional, Inst. de Geoci.
Ci. Ex., Univ. Est. Paulista, Rio Claro, SP (1985).

[9] S.R. Christofoletti, "Estudos mineralógicos, químicos e textural das rochas sedimentares da Formação Corumbataí Jazida Cruzeiro e suas implicações nos processos e produtos cerâmicos", Diss. Mestrado Geologia Regional, Inst. Geoci. Ci. Ex., Univ. Est. Paulista, Rio Claro, SP (1999).

[10] L.A. Gaspar Jr., "Estudo mineralógico, químico e textural das rochas sedimentares da Formação Corumbataí (Jazida Peruchi) e suas implicações como matéria prima para cerâmica vermelha", Diss. Mestrado Geologia Regional, Inst. Geoci. Ci. Ex., Univ. Est. Paulista, Rio Claro, SP (1999).

[11] C.D. Roveri, "Petrologia aplicada da Formação Corumbataí (Região de Rio Claro, SP) e produtos cerâmicos", Tese Dr. Geociências, Inst. Geoci. Ci. Ex., Univ. Est. Paulista, Rio Claro, SP (2010).

[12] A. Zanardo, M.M.T. Moreno, C.D. Roveri, A.C. de A.Prado, M.R. Motta, J.F.M. Masson, L. Ibrahim,"Mineralogical and textural characteristics of the raw material used in dry milling in the Ceramic Pole of Santa Gertrudes-Brazil”, In IX World Cong. Ceramic Tile Quality - Qualicer, Castellón, Espanha, 3 (2006) 181-184.

[13] ABNT, Associação Brasileira de Normas Técnicas, Placas Cerâmicas para Revestimentos, Especificações e Métodos de Ensaio (1997).

(Rec. 06/07/2015, Rev. 21/08/2015, 31/08/2015, Ac. $02 / 10 / 2015)$ 